\title{
Molecular markers in well-differentiated thyroid cancer
}

\author{
Anil K. D'Cruz ${ }^{1} \cdot$ Richa Vaish ${ }^{1} \cdot$ Abhishek Vaidya ${ }^{2,3} \cdot$ lain J. Nixon ${ }^{4} \cdot$ Michelle D. Williams ${ }^{5}$. Vincent Vander Poorten ${ }^{6}$. \\ Fernando López ${ }^{7}$. Peter Angelos ${ }^{8} \cdot$ Ashok R. Shaha $^{9} \cdot$ Avi Khafif $^{10}$. Alena Skalova ${ }^{11}$. Alessandra Rinaldo ${ }^{12}$. \\ Jennifer L. Hunt ${ }^{13} \cdot$ Alfio Ferlito $^{14}$
}

Received: 24 January 2018 / Accepted: 19 March 2018 / Published online: 6 April 2018

(c) Springer-Verlag GmbH Germany, part of Springer Nature 2018

\begin{abstract}
Purpose Thyroid nodules are of common occurrence in the general population. About a fourth of these nodules are indeterminate on aspiration cytology placing many a patient at risk of unwanted surgery. The purpose of this review is to discuss various molecular markers described to date and place their role in proper perspective. This review covers the fundamental role of the signaling pathways and genetic changes involved in thyroid carcinogenesis. The current literature on the prognostic significance of these markers is also described.

Methods PubMed was used to search relevant articles. The key terms "thyroid nodules", "thyroid cancer papillary", "carcinoma papillary follicular", "carcinoma papillary", "adenocarcinoma follicular" were searched in MeSH, and "molecular markers", "molecular testing", mutation, BRAF, RAS, RET/PTC, PAX 8, miRNA, NIFTP in title and abstract fields. Multiple combinations were done and a group of experts in the subject from the International Head and Neck Scientific Group extracted the relevant articles and formulated the review.

Results There has been considerable progress in the understanding of thyroid carcinogenesis and the emergence of numerous molecular markers in the recent years with potential to be used in the diagnostic algorithm of these nodules. However, their precise role in routine clinical practice continues to be a contentious issue. Majority of the studies in this context are retrospective and impact of these mutations is not independent of other prognostic factors making the interpretation difficult. Conclusion The prevalence of these mutations in thyroid nodule is high and it is a continuously evolving field. Clinicians should stay informed as recommendation on the use of these markers is expected to evolve.
\end{abstract}

Keywords Thyroid neoplasm/diagnosis · Thyroid neoplasm/genetics · Carcinoma, papillary · Adenocarcinoma, follicular · NIFTP · miRNA

This article was written by members and invitees of the International Head and Neck Scientific Group (http://www.IHNSG .com).

Anil K. D'Cruz

docdcruz@gmail.com

1 Head Neck Services, Tata Memorial Hospital, Parel, Mumbai 400012, India

2 National Cancer Institute, Nagpur, India

3 NKPSIMS, Nagpur, India

4 Departments of Surgery and Otolaryngology, Head and Neck Surgery, Edinburgh University, Edinburgh, UK

5 Department of Pathology, The University of Texas MD Anderson Cancer Center, Houston, TX, USA

6 Otorhinolaryngology-Head and Neck Surgery and Department of Oncology, Section Head and Neck Oncology, University Hospitals Leuven, KU Leuven, Leuven, Belgium
7 Department of Otolaryngology, Hospital Universitario Central de Asturias, IUOPA, University of Oviedo, CIBERONC, Oviedo, Spain

8 Department of Surgery and Surgical Ethics, The University of Chicago Medicine, Chicago, IL, USA

9 Head and Neck Service, Memorial Sloan-Kettering Cancer Center, New York, NY, USA

10 Head and Neck Surgery and Oncology Unit, A.R.M. Center for Advanced Otolaryngology Head and Neck Surgery, Assuta Medical Center, Tel Aviv, Israel

11 Department of Pathology, Charles University, Faculty of Medicine in Plzen, Plzen, Czech Republic

12 University of Udine School of Medicine, Padua, Italy

13 Department of Pathology, University of Arkansas for Medical Sciences, Little Rock, AR, USA

14 International Head and Neck Scientific Group, Padua, Italy 


\section{Introduction}

The prevalence of thyroid nodules ranges from $4-10 \%$ in the general population. This prevalence substantially increases to $50-70 \%$ when ultrasound is used for detection [1]. There has been a worldwide thyroid epidemic with 470,000 women and 90,000 men being over-diagnosed during the last 2 decades [2]. Though the disease-specific mortality of thyroid cancer is low, unwarranted or inadequate surgery is associated with increased morbidity making proper management important. Guidelines recommend fine needle aspiration cytology (FNA) for assessment of thyroid nodules [3]. However, it has limitations including indeterminate results and high inter-observer variability. The FNA is reported as indeterminate in around $25 \%$ of cases where the rate of malignancy ranges from 14 to $48 \%$ [4, 5]. It is important to identify malignant cases accurately to avoid unnecessary surgeries in those with benign nodules and target necessary therapy at those who require it.

The extent of surgery required for early tumours is controversial [3]. In part, this is due to an inability to determine the precise biology of tumours based on ultrasound and cytological features. There is a plausible need to take into consideration the biological aggressiveness of the tumour to refine surgical planning.

Recent years have witnessed considerable progress in the understanding of molecular changes underlying thyroid carcinogenesis. Molecular analysis is an emerging field and may enhance the prediction of both benignity and malignancy in thyroid cytology samples, increasing the total accuracy in cases when cytology is combined with molecular testing. Furthermore, molecular analysis is now formally included as an option for further evaluation of indeterminate cytology [3]. Despite the potential value in adults, molecular testing is not recommended in the pediatric population [6].

This review focuses on the current knowledge about molecular pathogenesis and markers of Differentiated Thyroid Cancer (DTC); their diagnostic and prognostic significance. We look at the fundamental role of signalling pathways involved in thyroid cancer and the genetic-epigenetic changes at the core of these pathways. Further, we consider molecular markers and tests developed thereof, their current role and evidence in diagnosis and prognosis in thyroid cancers.

\section{Molecular pathogenesis of differentiated thyroid cancers}

A large body of research in recent years has helped to improve our knowledge about molecular pathogenesis of thyroid cancers. This work has led to identification of specific molecular derangements and putative molecular pathways. More recently, The Thyroid Cancer Genome Atlas (TCGA) has performed a comprehensive analysis of Papillary Thyroid cancers (PTC) using multiple techniques including next generation sequencing. This has led to identification of specific abnormalities of significance in $96 \%$ of tumours [7].

\section{BRAF mutations and mitogen-activated protein kinase (MAPK) pathway}

BRAF is a serine-threonine kinase, belonging to family of RAF proteins, which are effectors of MAPK pathway. The MAPK is an intracellular signalling pathway that has a core function in cell growth, proliferation, apoptosis and differentiation; $B R A F$ alterations potently activate this pathway.

One of the most important gene mutations that play an important role in thyroid carcinogenesis and that has been most extensively studied involves $B R A F$ [8]. A point mutation (T1799A) causes V600E amino acid substitution in the BRAF protein, which is one of the common mutations that constitutively activate serine-threonine kinase. The frequency of this point mutation can be as high as $90 \%$ but is seen on an average in $45 \%$ of PTC [9]. BRAF mutations are not identified in benign thyroid nodules.

\section{RAS mutations and phosphoinositide 3 kinase-AKT (PI3-AKT) pathway}

RAS mutations are the second most common mutations in DTCs [9]. RAS in active state is bound to GTP and has intrinsic GTPase activity that converts GTP to GDP thus inactivating it. Mutation results in loss of this GTPase activity leading to its constitutive activation. Of the 3 isoformsHRAS, KRAS, NRAS, the most common mutations in thyroid cancers are in NRAS. Though RAS can activate both the MAPK and PI3-AKT pathway, the latter appears to be preferentially activated by the mutated RAS [9]. The PI3KAKT pathway has a predominant role in follicular patterned neoplasm including follicular thyroid carcinoma (FTC), wherein it promotes invasiveness and metastases. However, this appears to be an early mutational event having been identified in a portion of follicular adenomas also.

\section{RET/PTC rearrangement}

The $R E T$ proto-oncogene encodes a cell membrane receptor tyrosine kinase. RET is highly expressed in parafollicular C cells. It is usually not expressed in follicular cells, but it can be activated by chromosomal rearrangement: the RET/PTC translocation. This occurs due to genetic recombination between $3^{\prime}$ tyrosine kinase of RET and $5^{\prime}$ portion 
of a partner gene. The translocation constitutively activates tyrosine kinase activity of RET. RET/PTC activates both the MAPK and PI3-AKT pathways [9]. There are more than 10 types of this translocation, and the most common are RET/PTCl and RET/PTC3 [9-11].

Together RET/PTCl and RET/PTC3 rearrangements account over $80 \%$ of DTC $[12,13]$. RET/PTCl results in better differentiated PTC; whereas RET/PTC3 is more specifically observed in radiation-induced solid-follicular PTC [14]. RET/PTC related subtypes show more regional metastasis [15]. RET/PTC carcinogenesis follows increased expression and phosphorylation of epidermal growth factor receptor (EGFR) and can be potentially targeted by EGFR related tyrosine kinase inhibitors (TKIs) [16].

\section{PAX8/PPARY rearrangement}

PAX8/PPAR $\gamma$ rearrangement is caused by $(2 ; 3)(\mathrm{q} 13 ; \mathrm{p} 25)$ translocation that leads to fusion between the $P A X 8$ gene and the peroxisome proliferator-activated receptor- $\gamma(P P A R \gamma)$ gene [17]. PAX8/PPAR $\gamma$ has an inactivating effect on the wildtype tumour suppressor $P P A R \gamma$ and also transactivates certain $P A X 8$ responsive genes [9]. This translocation occurs in about $30-60 \%$ of FTC $[9,18,19]$ and also in $38 \%$ of follicular variant of papillary thyroid cancer (FVPTC) [19]. There is no overlap between PAX8/PPAR $\gamma$ and $R A S$ mutations in the same tumour [20], suggesting that FTCs may develop via 2 distinct pathways.

\section{Other genetic alterations}

Other genetic alterations are also involved in thyroid carcinogenesis. Mutations in the tumour suppressor gene PTEN activates the PI3K-AKT pathway, and forms the genetic basis of thyroid cancer in Cowden's disease [9]. TRK rearrangements may be found in less than 5\% of PTC [20]. An important recent discovery is of the human Telomerase Reverse Transcriptase (TERT) promoter gene mutations. This represents a newly discovered mechanism, by which cells acquire telomerase activity; these mutations have been found in aggressive thyroid cancers [21]. Less commonly p53, APC mutations (FAP), $A L K$ translocations, EIFIAX, etc. have also been described [22].

MicroRNAs (miRNAs) are short endogenous non-coding RNAs which regulate gene expression at mRNA post-transcriptional level in proliferation, apoptosis, and differentiation. Deregulation of miRNA expression is believed to be an important regulator of tumour development and progression [23]. Expression of miRNAs differs between PTC and benign thyroid lesions which may have a diagnostic implication in thyroid FNA and surgical pathology. In PTCs, there is a significant increase in miRNA (miR)-221, -222 and $-181 \mathrm{~b}$ as compared to normal thyroid. In particular, miR 221 over expression may have a critical role in thyroid cancers [24].
In a study, it was observed the seven miRNAs: miR-187, miR-221, miR-222, miR-146b, miR-155, miR-224 AND miR-197 was most consistently over expressed in follicular cell-derived carcinomas. However, their expression varied significantly between individual tumour types. When at least one miRNA was over expressed more than twofold, the sensitivity, specificity, and accuracy for malignancy detection were 100,94 , and $95 \%$ respectively [25].

\section{Molecular markers for diagnosis}

Understanding the molecular signatures of thyroid neoplasia has opened up new avenues to diagnosis of thyroid malignancies beyond cytologic classification; this is likely to have potential in nodules which are indeterminate on FNA. The Bethesda Reporting System for Thyroid Cytology (TBRSTC) is widely employed for reporting the outcome of FNA [26]. In 15-30\% cases, FNA cannot ascertain if the nodule is benign or malignant/ suspiciously malignant [27]. This includes Bethesda categories III, Atypia of Unknown Significance (AUS)/Follicular Lesion of Unknown Significance (FLUS) and IV, Follicular Neoplasm (FN). The risk of malignancy (ROM) in these categories varies from 5-15\% (category III) to $15-30 \%$ (category IV) [26]. This represents a challenge to physicians, since this ROM is too high to ignore. Performing 'diagnostic surgery' to obtain histopathology would be unnecessary in $70-80 \%$ of cases. In addition, there would be a need to perform an additional 'completion surgery' in nodules which are found to be 'highrisk' on pathology. A large body of work has focussed on the application of molecular alterations detection for improving the pre-operative diagnosis of thyroid cancers.

Recently non-invasive encapsulated follicular variant of PTC (FVPTC) has been classified as non-cancer entity with a revised nomenclature, non-invasive follicular neoplasm with papillary-like nuclei (NIFTP) [28, 29]. This poses a challenge to diagnostic accuracy of thyroid nodules further [30]. Studies have shown that these tumours are mainly classified as indeterminate on FNA [31]. With the reclassification of these tumours as non-malignant, there is a drop in ROM in the indeterminate categories. In a recent update taking this into account the ROM has shown a shift for category III from 10-30 to 6-18\%, category IV from $25-40$ to $10-40 \%$ and category $\mathrm{V}$ from $50-75$ to $45-60 \%$ [32]. Although this shift in nomenclature results in a drop in ROM, as NIFTP is still viewed as a neoplasm with frequent molecular alterations in $R A S$, lobectomy is still indicated for diagnosis and treatment.

To date, molecular elucidation in differentiated thyroid cancer (DTC) has found its main application in diagnosis; with several retrospective and prospective studies showing that the diagnostic accuracy of FNA can be significantly 
improved using molecular detection. We now review the genetic alterations that have shown diagnostic potential in FNA samples.

\section{BRAF}

$B R A F \mathrm{~V} 600 \mathrm{E}$ mutation has been the most extensively studied biomarker in FNA specimens. BRAF mutations are the most common genetic anomaly in papillary thyroid cancer (PTC), and are seen in about $45 \%$ of PTCs [9]. These are more common in sporadic PTCs, than in pediatric and radiation-induced PTCs [13]. BRAF mutations are rare in follicular thyroid cancer (FTC) and do not occur in benign thyroid nodules. Hence $B R A F \mathrm{~V} 600 \mathrm{E}$ mutation is a reasonably specific marker for PTC; however, this is the same group of patients where cytologic classification excels [12]. A review of 18 studies looking at $B R A F$ testing in thyroid FNA samples showed that the rate of malignancy in $B R A F$-positive nodules was $99.8 \%$ [33]. Moreover, it has been reported that 15\%-39\% of BRAF-positive FNA samples were indeterminate or non-diagnostic; thus proving diagnostic utility of $B R A F$ in indeterminate cytology. However, this utility of $B R A F$ as a diagnostic marker for indeterminate nodules is limited by its low sensitivity for malignancy [34]. Further $B R A F$ V600E is infrequent in FNA of indeterminate nodules $(<10 \%)$ which are typically follicular patterned neoplasms [35]. Nevertheless, irrespective of Bethesda category, when FNA testing reveals $B R A F \mathrm{~V} 600 \mathrm{E}$, a diagnosis of thyroid cancer should be strongly suspected, though rare false positive cases have been reported [36]. Although $B R A F$ V600E is the most commonly studied mutation, BRAF 599 and 601 have also been described which are seen in follicular patterned tumours. Translocations involving BRAF have also been documented.

\section{RAS}

$R A S$ mutations are seen in 40-50\% of FTC [20, 37] and in $10-20 \%$ of PTC [20]; most of which are FVPTC [38, 39]. However, RAS mutations are not specific for malignancy, and are also seen in $20-40 \%$ of follicular adenomas and are the predominant mutations in NIFTP [20]. This limits the utility of $R A S$ as a sole diagnostic marker of malignancy.

In a cohort of 199 thyroid carcinomas that underwent molecular characterisation 27 were $R A S$ mutation positive. Of these 20 were FVPTC, of which 16 would now be called NIFTP. Additionally, 59\% of RAS mutation positive carcinomas would now be classified as NIFTP [40]. These tumours are reported as suspicious by Afirma and constitute a significant proportion of carcinomas detected [41]. With the recent reclassification of NIFTP $[28,29]$ it is likely that the specificity of this molecular marker for carcinoma may decline. Nevertheless, it is argued that though not $100 \%$ specific,
$R A S$ detection implies a neoplasm for which surgical management, lobectomy only allows for further diagnosis and definitive treatment. The risk of malignancy in RAS FNA nodules awaits recalibration for incidence of true carcinomas/malignancies versus neoplasm (adenoma or NIFTP) secondary to the recent shift in classification.

\section{RET/PTC}

RET/PTC translocations are found in $15 \%-20 \%$ of sporadic adult PTC, but only in $6.8 \%$ in data from TCGA [7, 33, $36,42]$. They are more common in radiation-induced and pediatric PTCs $[10,13,43]$. RET/PTCl is the most common rearrangement type, seen in $60-70 \%$ of all cases [20]. In a retrospective study $R E T / P T C$ identified malignancy in $60 \%$ of indeterminate nodules with $0 \%$ false positive rate [44]. The results confirm that $R E T / P T C$ is a highly specific biomarker for the diagnosis of PTC. However, RET/PTC rearrangements in benign nodules remains debated [45]. The presence of these translocations implies risk of malignancy warranting further diagnostic characterization.

\section{Panel testing}

From the foregoing discussion, it can be inferred that the use of individual diagnostic markers has insufficient sensitivity and diagnostic accuracy. Hence efforts were directed towards the development of a panel of molecular markers and alterations for evaluation in FNAs. One initial approach was the development of a panel of mutations including $B R A F, R A S$, $R E T / P T C$ and PAX8/PPAR $\gamma$. This panel was first studied in 2 independent studies, and then in a large prospective study of indeterminate FNAs. The rate of malignancy in this study was $24 \%$, which is important to note as the rate of malignancy in the population being tested will affect the subsequent determinations. For indeterminate FNA, the panel had sensitivity $61 \%$, negative predictive value (NPV) $89 \%$, specificity 98\%, and positive predictive value (PPV) $89 \%$ [35]. Thus, the main impact of this panel was a rule in test by which the PPV and specificity for malignancy allowed for triaging patients to surgery when a mutation was identified.

Another approach was based on the measurement of mRNA expression. This gene expression classifier (GEC) yielded a high NPV (93\%) that reduced the ROM to about $5 \%$ in GEC benign nodules similar to the risk in a benign cytology FNA [46]. Thus, utilizing this method as 'rule out' could aid in triaging patients at low-risk for malignancy, without proceeding to surgical evaluation. Moreover, the advancement of next generation sequencing to allow for numerous loci analysis on minimal quantity of nucleic acids, further enhanced integrations of thyroid FNAs to methods currently clinically available. 


\section{Concept of 'rule in' and 'rule out'}

The choice of Molecular Test/Panel to be used broadly falls into two groups: (1) whether a test can 'rule in' malignancy (the likelihood that the nodule is malignant); or 'rule out' malignancy (the likelihood that the nodule is benign); and (2) depends on the performance factors of each test, the sensitivity, specificity, PPV and NPV.

A highly sensitive test is one which is usually positive in the presence of disease. Thus, a negative result of a highly sensitive test is associated with near surety of the absence of disease and conclusively rules out a condition. A highly specific test is one which is usually negative in the absence of disease. A positive result of a highly specific test is valuable in ruling in the presence of disease. In addition, the negative and positive predictive values of a test are directly proportional to its sensitivity and specificity, respectively, as well as to the prevalence of malignancy in the population being tested.

Of all the indeterminate nodules that undergo diagnostic surgery, two-thirds prove to be benign [4]. Thus, for the AUS, and FLUS and follicular neoplasm (FN) or Hurthle cell neoplasm categories (Bethesda III and IV), which harbour a 5-30\% risk of malignancy, an ideal molecular test to "rule out" malignancy would be one with high sensitivity and high NPV. Similarly, with regards to the suspicious for thyroid malignancy (Bethesda V) category, with a 60-75\% risk of malignancy, an ideal molecular test to "rule in" would be one with high specificity and high PPV. However, as no test is entirely sensitive, and Bethesda $\mathrm{V}$ has a high-rate of malignancy, this cohort warrants surgical evaluation of the nodule as ancillary tests cannot definitively exclude malignancy.

\section{Commercially available molecular tests}

There are a number of commercially available clinical tests to help further risk stratify indeterminate nodules to predict the presence or absence of malignancy (Table 1). Methods that classically test for the presence of gene point mutations are used as 'rule in test': gene point mutations (BRAF or $R A S)$ or gene rearrangements (RET/PTC, PAX8/PPAR $)$. The
Afirma GEC that checks for RNA expression is a classic example of 'rule out test'.

\section{Afirma gene expression classifier}

This is a microarray based test to analyse mRNA expression of 167 genes. A multicentre trial of GEC showed a high NPV of $95 \%$ and $94 \%$ for nodules in the AUS/FLUS and FN/SFN (suspicious for follicular neoplasm) categories (Bethesda categories III and IV), respectively [46]. Therefore, the ROM in these categories when the Afirma GEC test result indicated "benign nature" ranged from 5 to $6 \%$ and closely approached the NPV in thyroid FNAs diagnosed as benign. The GEC, however, has a low NPV value of $85 \%$ in Bethesda V 'Suspicious for malignancy' (thus representing a risk of cancer of 15\%). Hence the test is recommended only for categories III-IV. Further, the GEC has a low PPV value (37-38\%) [46]. This test is therefore useful as a 'rule out' test. If the diagnosis is 'benign' in the indeterminate category, the patient could be followed up clinically with no need for surgery. However, if the diagnosis is 'suspicious', the diagnosis remains indeterminate [47].

\section{miRInform test}

This test is based on analysis panel of 4 DNA mutations (BRAF, RAS, HRAS, and NRAS point mutations) and 3 RNA translocation fusion markers (RET/PTC1, RET/PTC3, and $P A X 8 / P P A R \gamma$ ) [47]. In a validation study the mutation positivity rate was significantly higher in the malignant cases (56\%) with highest rate reported in classical PTC (79\%) [48]. However, this test lacks sensitivity; it can therefore be used as a 'rule in' test to confirm malignancy in indeterminate or suspicious nodules on cytology.

\section{ThyGenX test}

This is a modified and currently offered version of the miRInform test. It uses next generation sequencing to identify more than 100 genetic alterations in 8 genes [47]. The test is applicable to only Bethesda Categories III-IV. Recently, a new test called ThraMIR is offered by the parent company,

Table 1 Overview of molecular diagnostic panel tests for thyroid FNA [47]

\begin{tabular}{lllll}
\hline & Afirma & ThyGenx & ThyroMIR & Thyroseq \\
\hline Characteristics & $\begin{array}{l}\text { High NPV and low PPV (rule } \\
\text { out) }\end{array}$ & $\begin{array}{l}\text { High PPV and low NPV (rule } \\
\text { in) }\end{array}$ & $\begin{array}{l}\text { High NPV and PPV when } \\
\text { combined with ThyGenx } \\
\text { (combined) } \\
\text { Methodology }\end{array}$ & $\begin{array}{c}\text { High NPV and PPV (com- } \\
\text { bined) }\end{array}$ \\
$\begin{array}{l}\text { Test results } \\
\text { mRNA gene expression }\end{array}$ & $\begin{array}{l}\text { Multiplex PCR } \\
\text { Benign/suspicious }\end{array}$ & $\begin{array}{l}\text { Specific gene mutation/trans- } \\
\text { location }\end{array}$ & $\begin{array}{l}\text { Negative/positive } \\
\text { Next generation sequencing }\end{array}$ & $\begin{array}{c}\text { Specific gene mutation/ } \\
\text { translocation }\end{array}$ \\
\hline
\end{tabular}

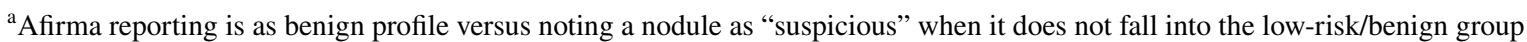


which is meant to be used in conjunction with ThyGenX when the result is negative. Using a combination of ThyGenX and ThyraMIR yielded a NPV and PPV of 94 and $74 \%$. When both test results were negative, the residual risk of cancer was 6\% [49].

\section{ThyroSeq test}

This is a next generation sequencing based gene mutation and fusion panel, targeting 284 mutational hot spots in 12 genes [50]. The assay identified mutations in $70 \%$ of PTC, $83 \%$ of FVPTC, $78 \%$ of FTC and 39\% of oncocytic follicular carcinoma. The test has high specificity and hence this can potentially be used as a 'rule in' test. A more recent version ThyroSeq v2 includes a more exhaustive panel of DNA and RNA alterations [point mutations in 13 genes and for 42 types of gene fusions: point mutations (AKT1, BRAF, NRAS, HRAS, KRAS, PTEN, TP53, TSHR, GNAS, CTNNB1, RET, $P I K 3 C A$, Primers for detecting mutations at the cytosine-tothymine 228 and 250 hotspots of the TERT gene promoter) and gene fusions (38 types of RET fusion genes including BRAF, NTRK1, NTRK3, ALK, PPARG, THADA to different partners)] and 8 genes as part of RNA panel to estimate the quantity of cells ( $P G K 1$ gene, TG, TTF1, NIS, KRT7, $C A L C A, P T H, K R T 20)$. A study using this version yielded a PPV of $83 \%$ and NPV of $96 \%$ [51] thus the ThyroSeq v2 may be used as both a 'rule out' and 'rule in' test. The latest version is ThyroSeq v3 which analyses 112 genes for point mutations, fusions, copy number changes, and expression levels that had been developed using a training set of 238 operated thyroid nodules. This was then validated on 175 FNA samples with known surgical follow-up. It showed a high sensitivity of $93.9 \%$, specificity of $89.4 \%$ and accuracy of $92.1 \%$ on the tissue training set. The sensitivity was $98.0 \%$, specificity $81.8 \%$, and accuracy $90.9 \%$ in FNA validation set [52].

\section{Rosetta GX assay}

This is a molecular microRNA-based assay which differentiates between benign and malignant nodule using reverse transcriptase-polymerase chain reaction (RT-PCR). In a study of over 800 FNA the results showed that the assay can be run on FNA slides with as little as $1 \%$ thyroid epithelial cells and with only $5 \mathrm{ng}$ of RNA [53]. The level of concordance was high between the laboratories and for the slides created from the same FNA pass. In another multicentric validation study on 189 samples, NPV, sensitivity and specificity was 91,85 and $72 \%$, respectively [54].

The new American Thyroid Association (ATA) Guidelines [3] recommend patient counselling about the potential benefits and limitations of molecular testing. The guidelines also state that since the long-term outcome data on use of molecular testing is still insufficient, it is debatable whether molecular testing should be used in routine practice for indeterminate cytology thyroid nodules.

\section{Molecular markers for prognosis}

Patient and tumour factors including age, tumour size, and presence and degree of extra thyroidal extension, number, size, location, extra nodal extension and distant metastases have been used to predict the risk of recurrence and mortality in DTC. Unfortunately, the majority of these are unavailable preoperatively. Postoperative ultrasound and thyroglobulin estimation are also used in clinical risk prediction [3]. More recently work has focused on molecular markers in predicting outcome for patients with thyroid cancer. Incorporation of these molecular markers into systems for risk stratification, which are available preoperatively, appears conceptually attractive in tailoring patient management in terms of initial extent of surgery, adjuvant therapy and postoperative medical management.

\section{BRAF}

Forty-eight to eighty percent of PTCs are associated with mutations. The most common mutation that occurs and studied extensively for its prognostic potential is $B R A F$, others being RAS, RET/PTC rearrangement and TERT $[55,56]$. In a recently published meta-analysis, the $B R A F$ mutation was present in $41.2 \%$ of all PTCs [57]. The prevalence of $B R A F$ mutation is higher in conventional PTC (51\%) when compared to FVPTC (24.1\%) and follicular carcinoma (1.4\%) [58]. There is geographical variation in the prevalence of $B R A F$ mutation with a relatively higher prevalence in Asia [59].

$B R A F$ is the most common mutation in PTC and most extensively studied for prognostication. BRAFV600E usually portends poor outcomes in PTC, and is associated with aggressive pathological features, increased rates of recurrence, loss of radioiodine avidity and treatment failures $[60$, 61]. In a multicentre study of 219 PTC, BRAF mutation was found to be significantly associated with extrathyroidal extension, lymph node metastasis, and advanced tumour stage. The mutation was also an independent predictor of recurrence. The mutation was associated with loss of radioiodine avidity and treatment failure in recurrent disease [61]. A meta-analysis of over 5000 patients also showed that the BRAF mutation was associated not only with an increased odds ratio of extrathyroidal extension, lymph node metastasis and advanced stage but also with a 2.14-fold increased risk of disease recurrence and persistence [56]. In another study $46 \%$ of those patients with central compartment lymph node metastasis had BRAF positivity, this 
being the only independent predictor of central compartment metastasis [62]. In a retrospective study of 1849 patients, cancer-related mortality was significantly higher in $B R A F$ positive patients; however, the association was not independent of other tumour features [63]. However, majority of these studies are retrospective and the impact of $B R A F$ positivity in some studies is not independent of other tumour features making the interpretation difficult.

These mutations are associated with aggressive tumour behaviour and poorer outcomes even in conventionally low-risk patients and papillary thyroid micro carcinoma (PTMC). In a comparative study of 1150 patients, it was seen that the frequency of a $B R A F$ mutation was similar in PTMC and PTC (65.6\% vs. 67.2\%) [64]. A BRAF mutation was associated with signs of higher aggressiveness, multifocality, extrathyroidal invasion, lateral neck compartment lymph node metastasis and advanced tumour stages III and IV in PTMC [65, 66]. A meta-analysis of over 3000 patients concluded that $B R A F$ positive PTMC is associated with tumour multifocality, extrathyroidal extension, lymph node metastasis and advanced stage [67]. BRAF mutations in low-risk cancer [intrathyroid tumours and without metastasis (T1-2N0M0)] was a poor prognostic factor for persistence of disease and these patients required radioiodine courses of a higher dose to obtain disease free status [68]. In another retrospective multicentre study, recurrence rates were significantly higher in $B R A F$ mutation positive PTC. This significant association of $B R A F$ mutation positivity with recurrence was also seen in conventionally low-risk disease stage, micro PTC and within various subtypes [69]. Therefore, there may also be an argument for treatment intensification for these low-risk patients with microcarcinomas who may otherwise be considered for observation in some settings.

Studies have shown that the detection of BRAF mutation had an independent correlation with worse outcome on multivariate analysis and mutation positive patients had lower percentage of survivors [70]. However, the long term impact of the mutation on survival has been challenged. At the median follow-up of 8 years there was no significant relationship between $B R A F$ mutation and recurrence-free survival and disease-specific survival (DSS) in another recent study [71]. A large meta-analysis reported that $B R A F$ mutations were significantly associated with poor diseasefree survival (DFS) and DSS at short/ medium follow-up (five or less than 5 years), however, this impact was lost at long term follow up of over 5 years [57].

The clinical application of BRAFV600E as prognostic marker is impaired by its low specificity. A meta-analysis showed an acceptable sensitivity (65\%), but a poor specificity for the prediction of recurrent disease with a PPV of only $25 \%$. Even though the mutation is associated with aggressive clinicopathological features, impact on survival in long term is not well established. Thus, the current role of mutated
$B R A F$ for risk stratification of PTC is limited. It is unlikely to be used in isolation, but only in a multivariable context, combined with other prognostic features [72]. While $B R A F$ may be associated with increased local recurrence as recognized by the ATA risk stratification [3] its role as an independent factor remains limited and its co-role with TERT promoter mutation may be the link to truly aggressive PTC, however prospective studies are needed.

\section{RAS}

RAS mutations are associated with tumour dedifferentiation and less favourable prognosis [20, 33, 73, 74]. Some studies have found a significant correlation between $R A S$ mutation and metastatic behaviour (bone metastases, in particular) and poor survival of follicular and papillary carcinomas [73, 74]. However, $R A S$ mutations may also be seen in encapsulated FVPTC, which is now considered non-cancer entity. Thus, RAS mutation in a thyroid nodule 'provides strong evidence for neoplasia, although it does not establish the diagnosis of malignancy'. At times, these mutations are found in adenomas; hence it is possible that $R A S$ mutated follicular adenomas are precursor lesions for FTC and FVPTC [20, 33].

\section{Others}

Clinically, RET/PTC positive PTC patients are younger and have classic papillary pathology and a high propensity of nodal metastases [15]. The prognostic implication of $R E T / P T C$ positive tumours are not clear. There is evidence that $R E T / P T C l$ is associated with more favourable behaviour in PTC; $[75,76]$ which contrasts with evidence that RET/PTC3 may portend dedifferentiation and more aggressive behaviour [76].

Clinically $P A X 8 / P P A R \gamma$ tumours are seen in younger patients, which are of smaller size with more frequent vascular invasion. Detection of PAX8/PPAR $\gamma$ in a follicular lesion should prompt the pathologist to perform a thorough search for vascular or capsular invasion [20].

Published studies have shown that TERT mutations are present in a small number of PTCs; however, these are subclonal [77]. In a meta-analysis, $B R A F$ mutations concomitant with TERT mutations were present in $6.2 \%$ and TERT alone in $4.2 \%$ of cases. TERT promoter mutations were associated with poor DFS and DSS and have been shown to be an independent predictor of recurrence and mortality [57]. TERT mutations were significantly associated with unfavourable survival both in short/ medium and long term followup. However, the majority of studies were retrospective and large prospective studies are needed.

Thus, the use of molecular markers for prognosis is plagued by a number of factors including the lack of 
specificity, variable reports regarding prognosis, and limited clinical utility. The use of molecular markers for prognosis to date has not found routine application in clinical practice. Current guidelines do not recommend the use of these markers for initial risk stratification. However, $B R A F$ and $T E R T$ promoter mutations are included in risk stratification for recurrence [3].

Another important factor of all the molecular tests is their high cost (\$1675-\$4875) [47]. For these to be commercially viable, results obtained should be able to offset the cost of treatment had the test not been offered. Yip et al. reported cost savings if the test was less than $\$ 870$. They concluded that molecular testing of indeterminate nodules can allow cost savings and improve patient care by providing an indication for total thyroidectomy when testing is positive [78]. However, these calculations may not hold true for regions outside the North America. In developing countries, the cost of the surgery may itself be lesser than the cost of the test. Further, the insurance coverage and a ceiling for such molecular tests are unlikely to be uniform.

\section{Conclusion}

The role of molecular markers in the management of patients with thyroid nodules and cancer is evolving. Although such tests have a potential role both in diagnosis and prognostication, the American Association of Clinical Endocrinologists (AACE) guidelines state that, "At present, molecular testing is meant to complement and not replace clinical judgment, sonographic assessment, and visual cytopathology interpretation. As molecular testing is new and advances in the field are regularly occurring, clinicians need to stay informed as recommendations for use within practice are expected to evolve" [79].

Funding None.

\section{Compliance with ethical standards}

Conflict of interest The authors declare that there is no competing interest.

Ethical approval This article does not contain any studies with human participants or animals performed by any of the authors.

\section{References}

1. Ezzat S, Sarti DA, Cain DR, Braunstein GD (1994) Thyroid incidentalomas. Prevalence by palpation and ultrasonography. Arch Intern Med 154(16):1838-1840
2. Vaccarella S, Franceschi S, Bray F, Wild CP, Plummer M, Dal Maso L (2016) Worldwide thyroid-cancer epidemic? The increasing impact of overdiagnosis. N Engl J Med 375(7):614-617

3. Haugen BR, Alexander EK, Bible KC et al (2016) 2015 American thyroid association management guidelines for adult patients with thyroid nodules and differentiated thyroid cancer: the American thyroid association guidelines task force on thyroid nodules and differentiated thyroid cancer. Thyroid 26(1):1-133

4. Wang C-CC, Friedman L, Kennedy GC et al (2011) A large multicenter correlation study of thyroid nodule cytopathology and histopathology. Thyroid 21(3):243-251

5. Nayar R, Ivanovic M (2009) The indeterminate thyroid fine-needle aspiration: experience from an academic center using terminology similar to that proposed in the 2007 National Cancer Institute Thyroid Fine Needle Aspiration State of the Science Conference. Cancer 117(3):195-202

6. Francis GL, Waguespack SG, Bauer AJ et al (2015) Management guidelines for children with thyroid nodules and differentiated thyroid cancer. Thyroid 25(7):716-759

7. Cancer Genome Atlas Research N (2014) Integrated genomic characterization of papillary thyroid carcinoma. Cell 159(3):676-690

8. Cohen Y, Xing M, Mambo E et al (2003) BRAF mutation in papillary thyroid carcinoma. J Natl Cancer Inst 95(8):625-627

9. Xing M (2013) Molecular pathogenesis and mechanisms of thyroid cancer. Nat Rev Cancer 13(3):184-199

10. Rabes HM, Demidchik EP, Sidorow JD et al (2000) Pattern of radiation-induced RET and NTRK1 rearrangements in 191 postchernobyl papillary thyroid carcinomas: biological, phenotypic, and clinical implications. Clin Cancer Res 6(3):1093-1103

11. Tallini G, Asa SL (2001) RET oncogene activation in papillary thyroid carcinoma. Adv Anat Pathol 8(6):345-354

12. Mehta V, Nikiforov YE, Ferris RL (2013) Use of molecular biomarkers in FNA specimens to personalize treatment for thyroid surgery. Head Neck 35(10):1499-1506

13. Vander Poorten V, Hens G, Delaere P (2013) Thyroid cancer in children and adolescents. Curr Opin Otolaryngol Head Neck Surg 21(2):135-142

14. Thomas GA, Bunnell H, Cook HA et al (1999) High prevalence of RET/PTC rearrangements in Ukrainian and Belarussian postChernobyl thyroid papillary carcinomas: a strong correlation between RET/PTC3 and the solid-follicular variant. J Clin Endocrinol Metab 84(11):4232-4238

15. Adeniran AJ, Zhu Z, Gandhi M et al (2006) Correlation between genetic alterations and microscopic features, clinical manifestations, and prognostic characteristics of thyroid papillary carcinomas. Am J Surg Pathol 30(2):216-222

16. Croyle M, Akeno N, Knauf JA et al (2008) RET/PTC-induced cell growth is mediated in part by epidermal growth factor receptor (EGFR) activation: evidence for molecular and functional interactions between RET and EGFR. Cancer Res 68(11):4183-4191

17. Kroll TG, Sarraf P, Pecciarini L et al (2000) PAX8-PPARgamma1 fusion oncogene in human thyroid carcinoma [corrected]. Science 289(5483): 1357-1360

18. Dwight T, Thoppe SR, Foukakis T et al (2003) Involvement of the PAX8/peroxisome proliferator-activated receptor gamma rearrangement in follicular thyroid tumors. J Clin Endocrinol Metab 88(9):4440-4445

19. Castro P, Rebocho AP, Soares RJ et al (2006) PAX8-PPARgamma rearrangement is frequently detected in the follicular variant of papillary thyroid carcinoma. J Clin Endocrinol Metab 91(1):213-220

20. Nikiforov YE (2011) Molecular diagnostics of thyroid tumors. Arch Pathol Lab Med 135(5):569-577

21. Liu X, Bishop J, Shan Y et al (2013) Highly prevalent TERT promoter mutations in aggressive thyroid cancers. Endocr Relat Cancer 20(4):603-610 
22. Acquaviva G, Visani M, Repaci A et al (2018) Molecular pathology of thyroid tumours of follicular cells: a review of genetic alterations and their clinicopathological relevance. Histopathology 72(1):6-31

23. Li X, Abdel-Mageed AB, Mondal D, Kandil E (2013) MicroRNA expression profiles in differentiated thyroid cancer, a review. Int J Clin Exp Med 6(1):74-80

24. Pallante P, Visone R, Ferracin M et al (2006) MicroRNA deregulation in human thyroid papillary carcinomas. Endocr Relat Cancer 13(2):497-508

25. Nikiforova MN, Tseng GC, Steward D, Diorio D, Nikiforov YE (2008) MicroRNA expression profiling of thyroid tumors: biological significance and diagnostic utility. J Clin Endocrinol Metab 93(5):1600-1608

26. Cibas ES, Ali SZ, Conference NCITFSotS (2009) The Bethesda system for reporting thyroid cytopathology. Am J Clin Pathol 132(5):658-665

27. Steward DL, Kloos RT (2014) Clinical diagnostic gene expression thyroid testing. Otolaryngol Clin North Am 47(4):573-593

28. Nikiforov YE, Seethala RR, Tallini G et al (2016) Nomenclature revision for encapsulated follicular variant of papillary thyroid carcinoma: a paradigm shift to reduce overtreatment of indolent tumors. JAMA Onco 12(8):1023-1029

29. Baloch ZW, Seethala RR, Faquin WC et al (2016) Noninvasive follicular thyroid neoplasm with papillary-like nuclear features (NIFTP): a changing paradigm in thyroid surgical pathology and implications for thyroid cytopathology. Cancer Cytopathol 124(9):616-620

30. Zhao L, Dias-Santagata D, Sadow PM, Faquin WC (2017) Cytological, molecular, and clinical features of noninvasive follicular thyroid neoplasm with papillary-like nuclear features versus invasive forms of follicular variant of papillary thyroid carcinoma. Cancer Cytopathol 125(5):323-331

31. Maletta F, Massa F, Torregrossa L et al (2016) Cytological features of "noninvasive follicular thyroid neoplasm with papillarylike nuclear features" and their correlation with tumor histology. Hum Pathol 54:134-142

32. Cibas ES, Ali SZ (2017) The 2017 Bethesda system for reporting thyroid cytopathology. Thyroid 27(11):1341-1346

33. Nikiforova MN, Nikiforov YE (2009) Molecular diagnostics and predictors in thyroid cancer. Thyroid 19(12):1351-1361

34. Nikiforov YE (2017) Role of molecular markers in thyroid nodule management: then and now. Endocr Pract 23(8):979-988

35. Nikiforov YE, Ohori NP, Hodak SP et al (2011) Impact of mutational testing on the diagnosis and management of patients with cytologically indeterminate thyroid nodules: a prospective analysis of 1056 FNA samples. J Clin Endocrinol Metab 96(11):3390-3397

36. Yip L, Ferris RL (2014) Clinical application of molecular testing of fine-needle aspiration specimens in thyroid nodules. Otolaryngol Clin North Am 47(4):557-571

37. Esapa CT, Johnson SJ, Kendall-Taylor P, Lennard TW, Harris PE (1999) Prevalence of Ras mutations in thyroid neoplasia. Clin Endocrinol (Oxf) 50(4):529-535

38. Zhu Z, Gandhi M, Nikiforova MN, Fischer AH, Nikiforov YE (2003) Molecular profile and clinical-pathologic features of the follicular variant of papillary thyroid carcinoma. An unusually high prevalence of ras mutations. Am J Clin Pathol 120(1):71-77

39. Gupta N, Dasyam AK, Carty SE et al (2013) RAS mutations in thyroid FNA specimens are highly predictive of predominantly low-risk follicular-pattern cancers. J Clin Endocrinol Metab 98(5):E914-22

40. Paulson VA, Shivdasani P, Angell TE et al (2017) Noninvasive follicular thyroid neoplasm with papillary-like nuclear features accounts for more than half of "carcinomas" harboring RAS mutations. Thyroid 27(4):506-511

41. Wong KS, Angell TE, Strickland KC et al (2016) Noninvasive follicular variant of papillary thyroid carcinoma and the Afirma gene-expression classifier. Thyroid 26(7):911-915

42. Zhu Z, Ciampi R, Nikiforova MN, Gandhi M, Nikiforov YE (2006) Prevalence of RET/PTC rearrangements in thyroid papillary carcinomas: effects of the detection methods and genetic heterogeneity. J Clin Endocrinol Metab 91(9):3603-3610

43. Fenton CL, Lukes Y, Nicholson D, Dinauer CA, Francis GL, Tuttle RM (2000) The ret/PTC mutations are common in sporadic papillary thyroid carcinoma of children and young adults. J Clin Endocrinol Metab 85(3):1170-1175

44. Cheung CC, Carydis B, Ezzat S, Bedard YC, Asa SL (2001) Analysis of ret/PTC gene rearrangements refines the fine needle aspiration diagnosis of thyroid cancer. J Clin Endocrinol Metab 86(5):2187-2190

45. Elisei R, Romei C, Vorontsova T et al (2001) RET/PTC rearrangements in thyroid nodules: studies in irradiated and not irradiated, malignant and benign thyroid lesions in children and adults. J Clin Endocrinol Metab 86(7):3211-3216

46. Alexander EK, Kennedy GC, Baloch ZW et al (2012) Preoperative diagnosis of benign thyroid nodules with indeterminate cytology. N Engl J Med 367(8):705-715

47. Zhang M, Lin O (2016) Molecular testing of thyroid nodules: a review of current available tests for fine-needle aspiration specimens. Arch Pathol Lab Med 140(12):1338-1344

48. Giordano TJ, Beaudenon-Huibregtse S, Shinde R et al (2014) Molecular testing for oncogenic gene mutations in thyroid lesions: a case-control validation study in 413 postsurgical specimens. Hum Pathol 45(7):1339-1347

49. Labourier E, Shifrin A, Busseniers AE et al (2015) Molecular testing for miRNA, mRNA, and DNA on fine-needle aspiration improves the preoperative diagnosis of thyroid nodules with indeterminate cytology. J Clin Endocrinol Metab 100(7):2743-2750

50. Nikiforova MN, Wald AI, Roy S, Durso MB, Nikiforov YE (2013) Targeted next generation sequencing panel (ThyroSeq) for detection of mutations in thyroid cancer. J Clin Endocrinol Metab 98(11):E1852-E1860

51. Nikiforov YE, Carty SE, Chiosea SI et al (2014) Highly accurate diagnosis of cancer in thyroid nodules with follicular neoplasm/ suspicious for a follicular neoplasm cytology by ThyroSeq v2 next-generation sequencing assay. Cancer 120(23):3627-3634

52. Nikiforova MN, Mercurio S, Wald AI et al (2018) Analytical performance of the ThyroSeq $\mathrm{v} 3$ genomic classifier for cancer diagnosis in thyroid nodules. Cancer. https://doi.org/10.1002/ cncr.31245

53. Benjamin H, Schnitzer-Perlman T, Shtabsky A et al (2016) Analytical validity of a microRNA-based assay for diagnosing indeterminate thyroid FNA smears from routinely prepared cytology slides. Cancer Cytopathol 124(10):711-721

54. Lithwick-Yanai G, Dromi N, Shtabsky A et al (2017) Multicentre validation of a microRNA-based assay for diagnosing indeterminate thyroid nodules utilising fine needle aspirate smears. J Clin Pathol 70(6):500-507

55. Lee J-H, Lee E-S, Kim Y-S (2007) Clinicopathologic significance of BRAF V600E mutation in papillary carcinomas of the thyroid: a meta-analysis. Cancer 110(1):38-46

56. Kim TH, Park YJ, Lim JA et al (2012) The association of the BRAF (V600E) mutation with prognostic factors and poor clinical outcome in papillary thyroid cancer: a meta-analysis. Cancer 118(7):1764-1773

57. Vuong HG, Duong UNP, Altibi AMAet al (2017) A meta-analysis of prognostic roles of molecular markers in papillary thyroid carcinoma. Endocr Connect 6(3):R8-R17 
58. Kebebew E, Weng J, Bauer J et al (2007) The prevalence and prognostic value of BRAF mutation in thyroid cancer. Ann Surg 246(3):466-470

59. Song YS, Lim JA, Park YJ (2015) Mutation profile of well-differentiated thyroid cancer in Asians. Endocrinology Metabolism 30(3):252-262

60. Lupi C, Giannini R, Ugolini C et al (2007) Association of BRAF V600E mutation with poor clinicopathological outcomes in 500 consecutive cases of papillary thyroid carcinoma. J Clin Endocrinol Metab 92(11):4085-4090

61. Xing M, Westra WH, Tufano RP et al (2005) BRAF mutation predicts a poorer clinical prognosis for papillary thyroid cancer. $\mathrm{J}$ Clin Endocrinol Metab 90(12):6373-6379

62. Howell GM, Nikiforova MN, Carty SE et al (2013) BRAF V600E mutation independently predicts central compartment lymph node metastasis in patients with papillary thyroid cancer. Ann Surg Oncol 20(1):47-52

63. Xing M, Alzahrani AS, Carson KA et al (2013) Association between BRAF V600E mutation and mortality in patients with papillary thyroid cancer. JAMA 309(14):1493-1501

64. Park YJ, Kim YA, Lee YJ et al (2010) Papillary microcarcinoma in comparison with larger papillary thyroid carcinoma in BRAF (V600E) mutation, clinicopathological features, and immunohistochemical findings. Head Neck 32(1):38-45

65. Lee X, Gao M, Ji Y et al (2009) Analysis of differential BRAF(V600E) mutational status in high aggressive papillary thyroid microcarcinoma. Ann Surg Oncol 16(2):240-245

66. Lin K-L, Wang O-C, Zhang X-H, Dai X-X, Hu X-Q, Qu J-M (2010) The BRAF mutation is predictive of aggressive clinicopathological characteristics in papillary thyroid microcarcinoma. Ann Surg Oncol 17(12):3294-3300

67. Li F, Chen G, Sheng C et al (2015) BRAFV600E mutation in papillary thyroid microcarcinoma: a meta-analysis. Endocr Relat Cancer 22(2):159-168

68. Elisei R, Viola D, Torregrossa L et al (2012) The BRAF(V600E) mutation is an independent, poor prognostic factor for the outcome of patients with low-risk intrathyroid papillary thyroid carcinoma: single-institution results from a large cohort study. J Clin Endocrinol Metab 97(12):4390-4398
69. Xing M, Alzahrani AS, Carson KA et al (2015) Association between BRAF V600E mutation and recurrence of papillary thyroid cancer. J Clin Oncol 33(1):42-50

70. Elisei R, Ugolini C, Viola D et al (2008) BRAF(V600E) mutation and outcome of patients with papillary thyroid carcinoma: a 15-year median follow-up study. J Clin Endocrinol Metab 93(10):3943-3949

71. Henke LE, Pfeifer JD, Ma C et al (2015) BRAF mutation is not predictive of long-term outcome in papillary thyroid carcinoma. Cancer Med 4(6):791-799

72. Tufano RP, Teixeira GV, Bishop J, Carson KA, Xing M (2012) BRAF mutation in papillary thyroid cancer and its value in tailoring initial treatment: a systematic review and meta-analysis. Medicine (Baltimore) 91(5):274-286

73. Basolo F, Pisaturo F, Pollina LE et al (2000) N-ras mutation in poorly differentiated thyroid carcinomas: correlation with bone metastases and inverse correlation to thyroglobulin expression. Thyroid 10(1):19-23

74. Garcia-Rostan G, Zhao H, Camp RL et al (2003) ras mutations are associated with aggressive tumor phenotypes and poor prognosis in thyroid cancer. J Clin Oncol 21(17):3226-3235

75. Mayr B, Brabant G, Goretzki P, Ruschoff J, Dietmaier W, Dralle H (1997) ret/PTC-1, -2, and -3 oncogene rearrangements in human thyroid carcinomas: implications for metastatic potential? J Clin Endocrinol Metab 82(4):1306-1307

76. Nikiforov YE (2002) RET/PTC rearrangement in thyroid tumors. Endocr Pathol Spring 13(1):3-16

77. Landa I, Ibrahimpasic T, Boucai L et al (2016) Genomic and transcriptomic hallmarks of poorly differentiated and anaplastic thyroid cancers. J Clin Invest 126(3):1052-1066

78. Yip L, Farris C, Kabaker AS et al (2012) Cost impact of molecular testing for indeterminate thyroid nodule fine-needle aspiration biopsies. J Clin Endocrinol Metab 97(6):1905-1912

79. Bernet V, Hupart KH, Parangi S, Woeber KA (2014) AACE/ ACE disease state commentary: molecular diagnostic testing of thyroid nodules with indeterminate cytopathology. Endocr Pract 20(4):360-363 